\title{
n-3 Polyunsaturated Fatty Acids and Cardiovascular Disease: Principles, Practices, Pitfalls, and Promises - A Contemporary Review
}

\author{
Richard Kones $^{\mathrm{a}}$ Scott Howell ${ }^{\mathrm{c}}$ Umme Rumana ${ }^{\mathrm{a}, \mathrm{b}}$ \\ ${ }^{a}$ The Cardiometabolic Research Institute, and b University of Texas Health Science Center Houston, Houston, TX, and \\ 'Department of Medicine, BMU School of Medicine, Winston-Salem, NC, USA
}

\section{Significance of the Study}

- There is immense interest in the cardiovascular use of n-3 polyunsaturated fatty acids (n-3 PUFA). Many potential roles in coronary disease, arrhythmias, heart failure (HF), and dyslipidemia have been identified, but clinical recommendations have been elusive. Hence, mechanistic and experimental evidence and reasons for prior mixed findings were reexamined. In 2 large, recent meta-analyses it was suggested that n-3 PUFA were associated with modest but statistically significant improvements in outcomes which might increase over time. In this review, a unique reinterpretation of evidence and the essentiality of using plasma or tissue n-3 PUFA levels are provided for future research.

\section{Keywords}

n-3 polyunsaturated fatty acids · Cardiovascular disease . Cardiovascular outcomes · Arrhythmias · Coronary heart disease - Myocardial infarction - Atherosclerosis . Endothelial function · Heart failure $\cdot$ Inflammation

\begin{abstract}
Amidst voluminous literature, inconsistencies and opposing results have confused rather than clarified cardiologists' ability to assess the potential benefits of $n-3$ polyunsaturated fatty acids ( $n-3$ PUFA). In perspective, there are common themes that emerge from n-3 PUFA studies, even as imperfect as they may be. The approach taken was to identify and unite these themes into a manageable, cohesive, evidencebased, yet useful synthesis. In all reviews and meta-analyses,
\end{abstract}

\section{KARGER}

E-Mail karger@karger.com www.karger.com/mpp

\section{(c) 2017 The Author(s) \\ Published by S. Karger AG, Basel \\ Karger \\ Open access}

This is an Open Access article licensed under the Creative Commons Attribution-NonCommercial-4.0 International License (CC BY-NC) (http://www.karger.com/Services/OpenAccessLicense), applicable to the online version of the article only. Usage and distribution for commercial purposes requires written permission. the selection of component studies and assumptions influences outcomes. This overarching principle must be combined with the totality of the data, particularly when evidence is incompletely understood and gaps in knowledge must be bridged. Both the older literature and the most recent rigorous meta-analyses indicate that n-3 PUFA are highly pleiotropic agents with many documented positive physiological effects. Concordance among preclinical, observational, randomized clinical trials and meta-analyses is impressive. These agents have modest, statistically significant benefits which accrue over time. Given their favorable safety profile, a risk reduction of about $10 \%$ justifies their potential use in cardiovascular disease.

(C) 2017 The Author(s)

Published by S. Karger AG, Basel
Richard Kones MD, FAHA, FESC, FRSM, FCCP, FAGS, FRSH, FRSB Cardiometabolic Research Institute 8181 Fannin Street, Building 3, Unit 314

Houston, TX 77054-2913 (USA)

E-Mail medkones@gmail.com 


\section{Introduction}

The debate about whether or not long-chain n-3 polyunsaturated fatty acids (n-3 PUFA) are evidence-based therapeutic agents in cardiovascular disease (CVD) has been argued for over half of a century. Morbidity and mortality from this disease, particularly coronary heart disease (CHD), is unacceptably high in every country, and even when state-of-the-art treatment is achieved, deadly events continue [1-3]. Additional data are also valuable for public health policymakers, since consumption of these agents is popular among $8 \%$ of the American population, and the global omega- 3 market amounts to USD 31.4 billion (KWD 9,460,820,000.00), as questions swirl about the wisdom of this practice [4]. Indeed, "fish oil" has consistently been one of the most popular subjects in the lay press during the past decades. For these reasons, it is appropriate to update the evidence supporting potential benefits of these molecules for individuals with and without heart disease.

\section{Background}

Interest in n-3 PUFA and CVD grew after a published association of the high n-3 PUFA intake of the Greenland Eskimo population and relatively low incidence of $\mathrm{CHD}$ [5]. This observation was remarkable in view of their traditional diet, which was relatively high in saturated fat and cholesterol, but low in fruit, vegetables, and complex carbohydrates. The theme was further developed by several authors, as in the work of Bang and Dyerberg [6]. Kromhout et al. [7] reported the inverse dose-response relation between fish consumption and adjusted CHD mortality over 20 years of follow-up. Individuals who ate $\geq 30$ g of fish per day exhibited less than half the mortality than those who did not, prompting the authors to conclude that consumption of just 1-2 fish servings per week could significantly affect CHD mortality [7]. In studying 20,551 participants in the US Physicians' Health Study followed for up to 11 years, Albert et al. [8] reported that in healthy men consuming $\geq 1$ serving of fish per week the relative risk (RR) for sudden cardiac death (SCD) was 0.48 (95\% CI 0.24-0.96; $p=0.04$ ) as compared with those who ate $<1$ serving/month ( $p$ for trend $=0.03$ ). Although intake was not associated with a lower risk of total myocardial infarction (MI), non-SCD, or total cardiovascular (CV) mortality, there was a significantly reduced association with total mortality. Albert et al. [9] extended this work by using n-3 PUFA blood levels in a prospective, nested case-control analysis in healthy men followed for approximately 17 years in the Physicians' Health Study for SCD as the first indication of CVD. Using 94 cases and 184 matched controls, baseline levels were inversely related to risk of SCD; the RR of SCD was significantly lower among those with levels in the 3rd quartile (adjusted RR $0.28,95 \%$ CI 0.09-0.87) and in the 4th quartile (adjusted RR 0.19, 95\% CI 0.05-0.71) as compared with the lower quartiles. Interestingly, adjustment for n-6 PUFA and saturated fats did not materially change this relationship, and adjustment for monounsaturated fat strengthened it. Harris et al. [10], combining 25 studies of n-3 PUFA levels in blood, plasma phospholipids, or tissue, reported that $\mathrm{CHD}$ events were inversely related with tissue levels of n-3 PUFA.

Since this earlier work, the literature examining the effects of n-3 PUFA upon CVD has exploded; the sophistication in experimental approach has improved, larger studies are available, and the scope of conditions has widened. At the same time, CV treatment has also progressed, and the standards against which n-3 PUFA effects are compared are different from those that existed in an earlier era. Space does not permit an exhaustive presentation of the voluminous data, but many reviews are available for the interested reader [11-22].

A relatively neglected area of omega-3 research was inattention to n-3 PUFA levels in both the preclinical and clinical experimental conditions explored. A recent review of 298 studies provided levels of fatty acids as plasma total lipids, plasma phospholipid, in erythrocytes and in whole blood [23]. For comparison, these data were converted to "relative weight percentages." The authors documented widespread differences in blood levels in adults geographically, but importantly, levels were extremely low in the Middle East. In view of the many clinicopathological correlations of such shortfalls in Middle Eastern countries, a higher level of awareness with a view toward intervention is in order. This is especially true since repleting low levels of n-3 PUFA may be more beneficial than simply giving more to individuals with full stores of the agent [24].

\section{Membrane and Electrophysiological Actions}

The cardioprotective actions attributed to n-3 PUFA could be due to the interaction of a number of pleiotropic effects, which included anti-inflammatory, antiatherogenic, antiarrhythmic, anti-inflammatory, antithrombotic/antiplatelet, antioxidant, antimitogenic, vasodilatory 
and hypotensive properties, associated improvements in endothelial function, and favorable lipid changes $[11,12$, 14]. Hence, n-3 PUFA was studied for reductions in allcause mortality, primordial, primary, and secondary prevention, acute atherothrombotic events, perisurgical protection, in arrhythmias, especially atrial fibrillation, type 2 diabetes (T2D), hypertension, HF, and other arterial and venous diseases. In several instances, these mechanisms have been linked and act synergistically.

A common theme in the study of beneficial effects was the approximate $50 \%$ reduction in SCD from relatively small amounts of fish: 1-2 servings/week or its equivalent in purified n-3 PUFA oil [8, 9]. Myocardial membranes may contain up to 2,000 lipids, and their fluidity is a function of the contents of PUFA, cholesterol, and phospholipids. There are highly assembled membrane areas in which saturated acyl tails of sphingolipids, cholesterol, and phospholipids coalesce to form structured microdomains called "lipid rafts" [25-27]. Substantial evidence now indicates that perturbations in such microdomains, typified by caveolae, react to physical and chemical changes, thereby altering cell signaling, intracellular transport, and ion channels [28]. Caveolae could be regarded as spatial organizers of signal transduction molecules. Minute changes in conformations or concentrations may therefore be accompanied by alterations in flexibility, compressibility, curvature, or thickness of the platforms integral to successful signaling or the completion of reactions. The partially enclosed flask shape of caveolae promotes a far higher concentration of ions inside it as compared with extracellular fluid, providing a fertile environment for the direct and indirect actions of lipids. Indeed, n-3 PUFA have potent effects upon ion channel proteins of nearly all types associated with lipid microdomains, including calcium regulatory proteins involved in additional processes, such as excitation-contraction coupling and inotropy [29-33].

Whether n-3 PUFA act directly or indirectly with the channel proteins remains unknown. When n-3 PUFA are given acutely and ion channel changes occur immediately, a direct action, or action in the membrane close to an ion channel, is more likely; longer-term administration allowing for incorporation into the sarcolemma may be associated with indirect effects [26, 27]. Marine (eicosapentaenoic acid [EPA; 20:5n-3], docosahexaenoic acid [DHA; 22:6n-3], and the metabolite docosapentaenoic acid [DPA; 22:5n-3]) and plant ( $\alpha$-linolenic acid [ALA; 18:3n-3]) n-3 PUFA influence many sodium, calcium, and potassium currents, but also modulate $\alpha$ - and $\beta$-adrenoreceptors. In addition, these agents are associ-

n-3 PUFA, CVD Risk, and Outcomes ated with beneficial effects on the autonomic system function, heart rate variability (HRV), and rate recovery after exercise. Intrinsic pacemaker rate alterations appear to be more important than rises in HRV, but HRV remains a predictor of mortality after MI and an index of cardiac health [34].

The n-3 PUFA given acutely block Kv1.5 and Kv11.1 ion channels at physiological concentrations [35], and depress the ultra-fast Nav1.5 [36] and L-type Ca channels [37]. These actions on the K-channels should lengthen the action potential duration (APD), but the actions on the Na- and Ca-channels should shorten the APD [35]. In addition, whether the net effect goes either way, the APD change may be antiarrhythmic or proarrhythmic, depending upon the cause of the arrhythmia. Prolonging the APD can be antiarrhythmic if the arrhythmia is due to reentry, but may also produce early afterdepolarizations (EAD) and Torsade de Pointes. On the other hand, shortening the APD may be antiarrhythmic if the arrhythmia is due to triggered EAD or delayed afterdepolarizations, but favors reentry by shortening the refractory period [37]. With respect to n-3 PUFA, these generalizations may not apply in the specific electrophysiological preparation, the method of application, the ani$\mathrm{mal}$, the dose, and the heart disease (substrate) under study. Furthermore, unlike EPA and DHA, the shorterchain plant ALA does not block Kv1.5 [35]. This said, the ion channel and antiadrenergic effects of n-3 PUFA noted above are similar to those of amiodarone, except that they do not widen QRS or prolong the QT interval, but they do prolong the APD, suppress Torsade de Pointes, and reverse triangulation, reverse use-dependence, instability, and dispersion (TRIaD) [36]. In fact, the reduction in SCD is greater with n-3 PUFA than with amiodarone in similar populations, while the adverse effects are not as limiting [36].

Even as compelling as some studies appear [35, 3739], the absence of recent strong confirmation of the decrease in SCD associated with n-3 PUFA administration is responsible for uncertainty and lack of recommendations in guidelines. In part, this may reflect methodological inhomogeneity and gaps in knowledge. In view of the recent interest in prescription n-3 PUFA, several studies in progress may clarify open questions. Remarkably, one must note that EPA and DHA are among the most studied of all therapeutic substances, and use of n-3 PUFA is strikingly prevalent, yet arrhythmic deaths associated with acute administration are extremely rare events, even when used during monitored parenteral and enteral feeding in critical care units [40]. Searching through several 
databases, finding documented examples of this phenomenon for study is difficult. Lands [41] expertly discussed the challenges in assigning weights to the: (i) confounding in existing studies concerning pharmacokinetics, pharmacodynamics, forms of n-3 PUFA, timescale factors, and topology, (ii) positive epidemiological and other evidence, (iii) particular use of n-PUFA in clinical settings, and (iv) safety.

The extent of both electrical and anatomic remodeling in heart muscle is a key determinant of the action of administered n-3 PUFA, which may partially account for minimal or inconsistent effects [34]. For example, in atrial fibrillation there are other variables, including cytokines and hypercoagulability involved in pathogenesis, in addition to the extent of pericardial fat and atrial fibrofatty infiltrates promoting electrical dissociation that become self-perpetuating. As was observed with drugs, remodeling beyond critical thresholds may drastically limit responsiveness. The GISSI-Prevenzione trial [42] with 11,323 participants provided the most recent confirmation of n-3 PUFA benefits post-MI in a large randomized trial, and again, effect sizes were greater than in comparable amiodarone studies $[36,43]$. The arm treated with the modest amount of $850 \mathrm{mg}$ of EPA+DHA daily enjoyed a $45 \%$ reduction in SCD, and 21 and $30 \%$ falls in total and CV mortality, respectively. As in other such studies, reductions in the rate of SCD account for much of the mortality benefit in primary and secondary prevention. Nonetheless, there were reports, largely theoretical, of potentially adverse n-3 PUFA actions in ischemic canine and porcine heart models [26,29-31]. It is important to note that in no human study in which n-3 PUFA levels were measured has this been confirmed. In contrast, the total number of all participants in studies examining SCD is now impressive [8-14, 34-37]. Finally, relying upon dietary recall and study designs that preclude comparison may account for some discrepancies [44, 45].

Another important factor in n-3 PUFA research is the rapidity, dose, preparation, and duration of administration. Experimentally, it may take months for full incorporation into membranes, and effects of acute and longer administration (days vs. weeks vs. months) may differ. However, as previously suggested, the full benefits of a high n-3 PUFA intake may require years to accrue. In a meta-analysis of 11 studies, 39,044 patients, including post-MI subjects, consumed an average dose of EPA/ DHA $1.8 \pm 1.2 \mathrm{~g} /$ day for a mean duration of $2.2 \pm 1.2$ years [46]. There was a significantly reduced risk of CV deaths (odds ratio [OR] 0.87, 95\% CI 0.79-0.95, $p=0.002$ ), SCD (OR $0.87,95 \%$ CI $0.76-0.99, p=0.04$ ), all-cause mortality
(OR 0.92, 95\% CI 0.85-0.99, $p=0.02$ ), and nonfatal CV events (OR 0.92, 95\% CI 0.85-0.99, $p=0.02$ ). Of note, the greatest mortality benefit was contributed by high-risk patients, and the improvement in nonfatal events occurred chiefly in the moderate-risk patients in the secondary prevention group. Although beyond the scope of this review, favorable metabolic actions of n-3 PUFA have persisted and/or resulted from sustained high intake over decades.

Based on some of the aforementioned, favorable data in primary prevention, about 7,125 CHD deaths could be prevented by sustained consumption of approximately $1 \mathrm{~g} /$ day of n-3 PUFA from farmed salmon per 100,000 individuals $[9,18,47]$. Since this number is offset by a small risk of deaths due to carcinogens consumed in the fish, this estimate for the equivalent in purified oils would be correspondingly greater.

\section{Clinical Studies of n-3 PUFA}

For CV outcomes in primary and secondary prevention and using either fish or n-3 PUFA oil, a surprisingly large number of participants have been included in a myriad of meta-analyses of randomized clinical trials (RCTs) and prospective cohort studies over the years [17, $46,48-57]$, in addition to innumerable individual studies. Collectively, these studies showed significant reductions in mortality and SCD [14, 18, 58]. One meta-analysis [19] with a large study population and number of events had low statistical significance $(p=0.0063)$, included many heterogeneous component studies, and did not employ an individual-patient meta-analysis. Of the studies [7, 59-66] that did not show positive results, the designs used had limitations; in some instances, these were considerable. In addition, several were underpowered to identify a statistically significant effect on cardiac mortality [7, 62-65]. The Risk and Prevention RCT of patients at high CVD risk but without prior MI was limited to low event rates, which necessitated a change in endpoints during the trial [66]. In others, the rigor in controlling n-3 PUFA intake varied from none to several grams daily, and often remained unknown [7, 51-58]. As mentioned, inaccurate self-reported data and poor adherence can be misleading and introduce appreciable bias which may remain ignored, particularly in meta-analyses [67]. This problem was also accompanied by variation in the background consumption of EPA+DHA in the populations studied, and additionally by the inclusion of plant n-3 ALA, which has different properties. Different doses used in each of 
the studies, ethnic variation in sensitivity to different $n-3$ PUFA properties, and even higher interindividual variability all impaired the credibility of the results. It is the preponderance and remarkable agreement from experimental work, data from preclinical trials of n-3 PUFA on risk factors, prospective observational studies, and fully powered RCTs that continue to drive interest.

A meta-analysis of 3 double-blind RCTs of CHD patients with implantable cardioverter-defibrillators examined the effects of n-3 PUFA versus placebo on spontaneous ventricular tachyarrhythmias; all of the 3 studies showed null results [55]. The rationale was that in CHD, triggered EADs and delayed afterdepolarizations and prolonged APDs are common proarrhythmogenic mechanisms, n-3 PUFA might suppress ventricular tachyarrhythmias, but in HF where the mechanism is predominantly reentry, the reverse would be expected. However, aside from the usual dose heterogeneity, underpowering left the meta-analysis unable to detect the risk reduction hypothesized, largely due to small sample sizes. However, these agents were previously found to be safe in HF patients [60]. GISSI-HF found that $1 \mathrm{~g}$ per day of $\mathrm{n}-3$ PUFA lowered all-cause mortality in New York Heart Association (NYHA) class IV HF patients [63]. While the absolute reduction in mortality for an average of 4 years was $1.8 \%$, the results were significant and achieved in patients who were being treated with multiple prescription drugs. The hypothesis followed that perhaps ventricular remodeling had been responsible. Prevention of ventricular hypertrophy in concert with improved cardiac dysfunction by $n-3$ PUFA has been documented in several animal preparations; postinfarction, a reorganization of junctional areas in association with changes in the protein connexin 43 , involved in growth and differentiation, have been documented $[68,69]$.

Nodari et al. [70] randomized nonischemic dilated cardiomyopathy HF patients with minimal symptoms to $2 \mathrm{~g}$ of n-3 PUFA or placebo. After 1 year, the n-3 PUFA group had a significant rise in left ventricular ejection fraction (LVEF), peak oxygen consumption $\left(\mathrm{VO}_{2}\right)$, and exercise duration, accompanied by a fall in mean NYHA functional class and rates of rehospitalization. While this study also had limitations, an editorial speculated about a greater effect if higher n-3 PUFA doses were used [71]. Use of n-3 PUFA was rated as level of evidence $B$ in the ACCF/AHA HF guidelines, which considered such therapy reasonable in patients with NYHA class II-IV symptoms and either HF with a reduced ejection fraction or HF with a preserved ejection fraction, to reduce both mortality and recurrent hospitalizations [72]. In a meta-analysis

n-3 PUFA, CVD Risk, and Outcomes of 7 trials with 825 chronic HF participants, treatment with n-3 PUFA significantly increased LVEF and reduced left ventricular end-systolic volume [73]. This effect was more pronounced in patients with nonischemic HF along with improvements in peak $\mathrm{VO}_{2}$ and NYHA functional classification. The degree of changes in these indices would correspond to a $6 \%$ lower risk of overall mortality, but the additional import is that, once again, n-3 PUFA was associated with improved left ventricular function and restorative remodeling. Reversal of adverse remodeling involving fibrosis-, hypertrophy-, and inflammationrelated genes were previously reported in a model of atrial cardiomyopathy [74].

Several other mechanisms contribute to the beneficial effects of n-3 PUFA in HF patients, which traditionally include a hypotensive action, reduction in collagen deposition, suppression of arrhythmias, and improvements in cardiac energetics. Omega-3 PUFAs could preserve cardiac mitochondrial function by stimulating the expression of proteins that improve cardiac lipid metabolism, lowering myocardial oxygen consumption, and raising mechanical efficiency. Endothelial function improves through the many pleiotropic properties of n-3 PUFA that are interrelated: increasing nitric oxide (NO) production, decreasing arterial stiffness, quelling inflammation, and molecular antiatherosclerotic mechanisms [75, 76]. For instance, not only is NO production important, but reducing plasma triglycerides (TG) may also modify arterial flexibility independently. When TG-rich lipoproteins (TRL) are hydrolyzed, remnant-like lipoprotein particles form, also contributing to endothelial dysfunction by impairing NO synthase; both mechanisms can be attenuated by n-3 PUFA. By raising levels of adiponectin, n-3 PUFA indirectly cause additional NO expression. n-3 PUFA also protect the endothelium against angiotensin II- and glucose-mediated injury, lower membrane arachidonic acid content, and displace ceramide, thereby opposing ceramide signaling that induces vasoconstriction mediated by thromboxane [77]. Insertion of EPA into membranes interrupts waves of peroxidation, protects low-density lipoprotein cholesterol (LDL-C), and lowers the level of small, dense LDL-C (sdLDL), which itself causes endothelial dysfunction. Inflammatory agonists can raise monocyte adhesion to the endothelium. This is reversed with EPA, but accentuated by arachidonic acid. EPA augments the bioavailability and function (migration and integration into tubules) of endothelial progenitor cells, i.e., stimulates neovasculogenesis. Inflammation and high sympathetic drive play a significant role in HF, and levels of C-reactive protein are among many proin- 
flammatory mediators that are suppressed by n-3 PUFA, along with a fall in nuclear factor-kappa B activity, TNF- $\alpha$, IL-1, IL-6, and oxidative stress [78, 79]. Another metaanalysis recently confirmed a rise in LVEF in dilated cardiomyopathy patients, but also emphasized 2 additional mechanisms of interest: decreased brain natriuretic peptide levels and serum norepinephrine levels [80].

In summary, several processes could be responsible for improved function of the failing heart observed after $n-3$ PUFA administration. One of many that need further research is gene modulation in 2 pathways, one involved with lipid transport and metabolism, and the other with the downregulation of extracellular and cardiac matrix remodeling, inflammation, and cell growth. One dose schedule that has been advocated for the early treatment of HF is $5 \mathrm{~g}$ per day for 30 days followed by a maintenance dose of $2 \mathrm{~g}$ per day of n-3 PUFA in the absence of contraindications [71].

\section{Role of Measuring n-3 PUFA Levels}

Measuring n-3 PUFA levels constitutes a major advance in the methodology of conducting clinical n-3 PUFA trials, which may minimize some sources of bias and decrease heterogeneity among studies. Both blood and tissue levels could facilitate comparisons of findings, especially if differences are due to poor adherence to dietary, supplement, and prescription n-3 PUFA instructions, when there is a variation in the content of supplements, adulteration and mislabeling, digestive or absorption issues, or varying bioavailability [81]. Not infrequently, patients will substitute an over-the-counter (OTC) brand for a prescription version without informing physicians; contents of commercial brands vary appreciably, and often patients will not understand that the total marine oil in a bottle is not the same as the actual weights listed. This challenge has greater relevance than appreciated: when OTC brands were tested for the ability to inhibit copper-induced oxidation of human sdLDL in vitro, $\geq 30$ other fatty acids were present [82]. In all, 10-14 different saturated species accounted for up to $36 \%$ of the total fatty acid content. A substantial percentage of these samples failed to inhibit sdLDL, likely due to saturated or peroxidized fats which remained undetected. Measurement of n-3 PUFA levels may not help identify this more complex type of error. More important than simply failing to inhibit sdLDL oxidation, the levels of saturated fat and peroxides were deemed sufficient to inflict harm themselves. Finally, OTC n-3 PUFA consumption is prevalent, and may not be revealed in intake questionnaires or interviews. Hence, even "control" patients may have higher background n-3 PUFA levels than expected, potentially confounding calculations of effect sizes between groups.

In the Danish Diet, Cancer and Health study of 57,053 healthy enrollees unselected for cardiac risk factors, a buttock adipose biopsy was taken at baseline, along with demographic and medical information [83]. Incident cases of acute coronary syndrome (ACS) were matched, and levels of EPA, DHA, and DPA in the tissue were determined. After a mean follow-up period of 7.6 years, there were 1,012 cases of ACS. A significant inverse monotone relationship between the total n-3 PUFA in the adipose tissue, which accurately reflects dietary n-3 PUFA consumed over the prior 1-3 years, and incidence of ACS was found in men. The same negative relationship held for DHA, but not for EPA or DPA. Fewer women than men participated in the study, and the number of events in women was smaller, probably attributable to the later age at which women suffer ACS. The levels of n-3 PUFA in adipose biopsies were lower than expected, and interindividual differences were not as great - characteristics that may or may not be unique. Such low levels may have impaired the detection of an association between n-3 PUFA and ACS.

In contrast with many prior n-3 PUFA-ACS studies, the association was primarily driven by a lower incidence of nonfatal ACS. The use of tissue levels in this study enabled high-quality exposure assessment to describe the relationship with outcomes, bypassing food frequency questionnaires, and the potential for recall bias. These results agree with prior studies showing that the EPA+DHA content of red blood cell (RBC) membranes, as a percent of total FAs, is lower in ACS patients compared to matched controls (20\% lower in this instance); EPA+DHA levels vary inversely with the OR for an ACS event [84]. Moreover, even in populations with high dietary consumption of n-3 PUFA, the ratios of EPA and DHA to arachidonic acid predict early ACS [85]. The "omega-3 index" (O3I), the sum of $\mathrm{RBC}(\mathrm{EPA}+\mathrm{DHA})$ as a percent of total fatty acids, is a common measurement which is inversely related to risk for SCD, ACS, all-cause mortality, and many of the pathophysiological biomarkers investigated [86]. Interestingly, the explained variability in the adjusted O3I was $73 \%$, with heritable (24\%), dietary intake $(25 \%)$, and supplements (15\%) as major components [86].

The importance of measuring actual levels of $n-3$ PUFA cannot be overemphasized; the strength of any preclinical or clinical association with these agents de- 
pends upon confirming the presence of a suitable intervention, and documented elevations in blood or tissue levels are reliable means of doing so. Simply assigning individuals to groups, supplying n-3 PUFA, or giving prescriptions to participants invites lack of rigor and impairs validity in trials.

\section{Recent Major Meta-Analyses}

\section{The Study by Del Gobbo et al. [87]}

In 19 studies (prospective [cohort, nested case-control] or retrospective) in which tissue or blood levels of n-3 PUFA were measured, 45,637 participants (mean age 59 years, $63 \%$ male) were followed for the development of incident CHD, nonfatal and fatal CHD, and the results were pooled [87]. Participants were excluded if they had a prior history of $\mathrm{CHD}$, angina, or coronary revascularization. There were 7,973 MIs leading to 2,781 deaths and 7,157 nonfatal MIs.

The meta-analysis included EPA, DHA, DPA (22:5n3 ), chiefly derived from endogenous metabolism, and plant ALA. For many CV actions, ALA is not as potent as EPA or DHA, but does have effects of its own. ALA can be converted to EPA, and EPA to DHA, but this process amounts to a few percent, less for DHA, and conversion is further compromised in cardiometabolic disease [88]. As is the case for EPA/DHA and their n- 6 counterparts in membranes, the n-3 and n- 6 series compete for 2 enzymes responsible for metabolism and transformation. Since the Western diet is high in n-6 PUFA, especially from vegetable oils, the ratio of n- 6 to n-3 intake has fallen from approximately $1: 1$ in Paleolithic man to the current value of approximately 1:20, with deleterious effects on health [89].

Each component study conducted standardized, individual-level analyses using harmonized models, exposures, outcomes, and covariates. n-3 PUFA were measured in total plasma, phospholipids, cholesterol esters, and in adipose tissue, and were recorded as the weight percentage of total fatty acids. In addition, heterogeneity was analyzed by age, gender, ethnicity, the presence of T2D, use of statins or aspirin, n-6 levels, and FADS desaturase genes $(\triangle 5$-desaturase gene [FADS1] catalyzes the conversion of $20: 4 n-3$ [eicosatetraenoic acid] to $20: 5 n-3$ [EPA], but also 20:3n-6 to 20:4n-6, arachidonic acid, in the $\mathrm{n}-6$ series; $\triangle 6$ desaturase [FADS2] assists with the 18 : $3 n-3$ [ALA] to $18: 4 n-3$ [stearidonic acid] and $24: 5 n-3$ [tetracosapentaenoic acid] to $24: 6 n-3$ [tetracosahexaenoic acid] conversions, which form DHA upon $\beta$-oxidation). Other covariates were age, body mass index, educational

n-3 PUFA, CVD Risk, and Outcomes level, smoking, physical activity, alcohol intake, T2D, and treated hypertension.

After adjustment, for every 1-standard deviation (SD) increase in the n-3 PUFA reported, there was a significant association with a decreased risk of fatal CHD (RR: ALA, 0.91 [95\% CI 0.84-0.98]; DPA, 0.90 [95\% CI 0.85-0.96], and DHA, 0.90 [95\% CI 0.84-0.96]). Of the 3 main n-3 PUFA, only DPA was linked to a lower risk of total CHD (RR 0.94, 95\% CI 0.90-0.99); there were no significant associations with nonfatal MI per $1 \mathrm{SD}$. The upper quintiles of EPA and DHA were associated with a reduced risk of nonfatal MI (RR 0.71 [95\% CI 0.56-0.90] vs. 0.87 [95\% CI 0.78-0.97]). The highest quintiles of DPA and DHA were associated with a significantly lower risk of fatal CHD (DPA [RR 0.76, 95\% CI 0.65-0.90] and DHA [RR $0.77,95 \%$ CI 0.64-0.89]).

This meta-analysis was unique in several respects. Results indicated that in vivo levels of ALA, DPA, and DHA were associated with a lower incidence of fatal CHD - a significant approximate $9 \%$ fall per 1 SD rise in these values. The population was diverse, not restricted, and effects were independent of age, sex, and n-6 PUFA (linoleic acid [18:2n-6] and arachidonic acid [20:4n-6]) levels. The variation was due only to dietary fish intake, avoiding limitations due to the use of self-reported amounts of fish or capsules consumed. The sample size was large, assuring a sufficient number of events for analysis. The number of component studies lowered the probability of publication bias, and inclusion criteria minimized reverse causation. Although some component studies used plasma levels, plasma phospholipids or cholesteryl esters, RBC levels, and others adipose tissue, comparing highest to lowest quintiles captured the essence of the effect, since there is good correlation between these biomarkers. Analysis was prespecified, and standardized definitions and modeling for all variables and outcomes were employed.

The data agree with many prior prospective cohort studies, RCTs, meta-analyses, and recent additional largescale contributions that increased n-3 PUFA intake is associated with a lower risk of fatal MI or major events $[8$, $14,16,17,58,90,91]$.

\section{The Study by Alexander et al. [91]}

From January 1947 through November 2015 a literature search was conducted to identify RCTs and prospective cohort studies relating EPA+DHA consumption and CHD outcomes [91]. Participants were nonhospitalized adults with and without CHD but free of serious noncardiac disease. The study was composed of 2 meta-analyses: in the first, 18 RCTs randomly assigned approximately 
93,000 participants to consume a specific amount of $n-3$ PUFA. The second analyzed 16 trials and approximately 732,000 participants, followed for CHD events over a long period of time.

For inclusion, CHD outcomes, specifically MI (fatal or nonfatal), angina, SCD, coronary death, and CHD incidence (for prospective cohort studies) were required, as well as hazard ratios (HR) or rate ratios of outcomes and measures of variance (i.e., 95\% CIs), or data to calculate these measures. For RCTs, a composite variable, "any CHD event," was defined as a combination of fatal or nonfatal MI, coronary death, SCD, and angina. For prospective analyses, "any CHD event" was considered to be the same, with the addition of CHD incidence.

Random-effects meta-analysis models were employed to derive summary RR estimates and 95\% CIs. Analysis was performed for larger samples of "any CHD event" and component, specific events. Heterogeneity was evaluated in subgroup and sensitivity analyses, and by metaregression. Dose-response was evaluated in either stratified dose or intake analyses. The Cochran Q was used to assess between-study statistical variation. Finally, 2 methods of assessing publication bias were used.

The meta-analysis of RCT data revealed that n-3 PUFA supplements in $\geq 1$ g per day doses (different forms) given for primary and secondary prevention were associated with a statistically insignificant $6 \%$ fall in CHD risk for any event (HR 0.94, 95\% CI 0.85-1.05). However, in the subgroup with TG levels $>150 \mathrm{mg} / \mathrm{dL}$, the CHD risk fell by $16 \%$ (HR $0.84,95 \%$ CI $0.72-0.98$ ), and in those with LDL-C levels $>130 \mathrm{mg} / \mathrm{dL}$ the risk fell by $14 \%$ (HR 0.86 , 95\% CI $0.76-0.98$ ). In the meta-analysis of prospective cohort studies, additional n-3 PUFA intake (all intake categories, follow-up range from 4.3 to 40 years) significantly reduced CHD risk of any event by $18 \%$ (HR 0.82 , 95\% CI 0.74-0.92).

The final data in these meta-analyses not only add further to the momentum of positive evidence favoring the cardioprotective effects of n-3 PUFA, but do so in a different, important direction. The current pandemics of obesity, T2D, and metabolic syndrome reflect molecular phenotypes with a high cardiometabolic risk [2,3]. Plasma TG $<100 \mathrm{mg} / \mathrm{dL}$ is generally considered an index of metabolic health, but up to $33 \%$ of the adult US population have levels in excess of $150 \mathrm{mg} / \mathrm{dL}$. Current guidelines do not recommend treatment for concentrations $<500 \mathrm{mg} / \mathrm{dL}$. Strong data using Mendelian randomization designed to minimize confounding and reverse causation now show that elevated TG levels are causes of low-grade inflammation, CHD, and all-cause mortality [92]. Further genetic studies have also clearly established TRLs as causes of CHD, ischemic stroke, and all-cause mortality [93]. As already mentioned, remnant cholesterol, i.e., the cholesterol content of particular TRLs called remnants (including chylomicron remnants, and very low-density lipoproteins and intermediate-density lipoproteins in the nonfasting state and in the fasting state) also cause CHD and lowgrade inflammation [94]. These relationships are independent from vascular damage attributable to LDL-C, are present in individuals without T2D or obesity, are substantial, and partially account for the residual risk remaining in patients being treated with evidence-based protocols [95-98]. In this respect it is appropriate to note that each additional $1 \mathrm{~g}$ per day of EPA-DHA consumed is associated with a fall in TG levels by $-5.9 \mathrm{mg} / \mathrm{dL}$ (95\% CI -2.5 to -9.3 ) [58]. Past meta-analyses did not stratify according to levels of TG, TRLs, or cholesterol remnants.

An editorial accompanying this study [91] acknowledged that it is the most comprehensive in the medical literature, and improvements in outcomes were impressive since they occur in statin-treated patients with no clinically important side effects. They too endorsed use of n-3 PUFA levels or the omega-3 index, a convenient and increasingly available tool $[16,23,86]$. Users should become familiar with reports in the various fatty acid profiles, with particular care in interpreting effects of the composition and properties of the n-3 PUFA administered, especially plant ALA, and the individual fatty acid levels reported. Due to the biochemical pathways involved, effects of ALA intake upon EPA levels will usually be minimal.

Updating a prior scientific statement [18], a recent American Heart Association Advisory survey [99] was limited in scope to large RCTs using supplements and major clinical CVD end-points, including diabetes. By definition, observational, mechanistic, physiological, or electrophysiological studies in humans, and those involving n-3 PUFA levels or fish, were excluded. Not surprisingly, in view of these restrictions, no recommendation could be made in several categories. However, on the basis of their deliberations, and comments that for many clinical situations "a lack of evidence of a benefit differs from evidence of a lack of effect," treatment was considered reasonable in 2 subgroups: (a) secondary prevention of SCD and CHD among patients with prevalent CHD (class IIa, benefit much stronger than risk; level of evidence high ["A"], multiple populations involved/more than $1 \mathrm{RCT} /$ meta-analysis of high-quality RCTS/ $\geq 1$ RCTs corroborated by high-quality registry studies), and (b) secondary prevention of outcomes in HF patients (9\% reduction in mortality and hospitalizations in HF pa- 
tients with a reduced ejection fraction; class IIa; level of evidence moderate ["B-R," $\geq 1$ or more RCTs or metaanalyses of moderate-quality RCTs]).

A large meta-analysis of 14 RCTs involving 71,899 participants compared cardiac mortality in an n-3 PUFA arm (pharmaceuticals and supplements for $\geq 6$ months) with a control arm [100]. There were 1,613 cardiac deaths $(4.48 \%)$ in the intervention arm and 1,746 cardiac deaths $(4.87 \%)$ in the control arm. Pooled RR estimates showed an $8.0 \%$ (95\% CI $1.6-13.9 \%, p=0.015)$ lower risk in the n-3 PUFA group versus the controls. In subset analyses there were numerically larger effects (12.9-29.1\% lower risks, all $p<0.05$ ) in subsets of RCTs with EPA+DHA dosages $>1$ g per day and higher risk samples (in secondary prevention, baseline mean or median $\mathrm{TG} \geq 1.69$ $\mathrm{mmol} / \mathrm{L}[150 \mathrm{mg} / \mathrm{dL}], \mathrm{LDL}-\mathrm{C} \geq 1.69 \mathrm{mmol} / \mathrm{L}[130 \mathrm{mg} /$ $\mathrm{dL}$ ], statin use $<40 \%$ of subjects). These data lend further credence to the AHA Science Advisory that n-3 PUFA treatment is reasonable in secondary prevention to prevent SCD and in those with HF [99]. This particular meta-analysis, however, suggests that patients at higher risk, as reflected by TG and LDL-C levels, benefit more from n-3 PUFA than others [101]. In comparing the results of meta-analyses, the choice of study design, which RCTs to include, and outcomes, clearly has a strong influence on result consistency, with effect sizes varying from 0.45 to 1.45 [101]. The data from the recent trials considered herein strongly support a significant modest improvement of n-3 PUFA therapy in CHD patients, which vary according to subgroups $[87,91,99,100]$.

Three relatively unaddressed issues have been raised regarding the available data and interpretation of results, namely n-3 PUFA levels, additional CV risk from circulating nontraditional atherogenic moieties, and optimal doses in subsets of patients. As mentioned above, in the AHA Advisory, studies using n-3 PUFA levels were deliberately excluded [99]. Yet the variation of levels within populations is striking [23]. In many instances, administering a fixed physiological dose of n-3 PUFA to a cohort in which background levels randomly vary may result in overlap between baseline levels and postintervention levels. In an RCT, this would translate to permitting the uncontrolled contamination of groups with an active agent prior to administering the same agent, and then claiming to study the interventional effect of the latter. It is also possible that increases in differences in background intake of n-3 PUFA, for instance due to a steep rise in supplement use, may account for decreases in effect sizes in clinical trials over the past 2 decades. Obviously, any statements made concerning the existence of an interventional effect or its size would be invalid. Use of n-3 PUFA levels would also identify nonresponders whose levels do not rise with exogenous dosing. Moreover, benefits of n-3 PUFA therapy are greater in patients with low baseline stores $[24,101]$. The inclusion of studies that rely upon self-recall of $n-3$ product use, while rejecting those that rely upon n-3 PUFA levels, is particularly concerning. In the future, perhaps knowledge of n-3 PUFA levels will be considered as an advantage, not as a confounder to be ignored $[14,23$, 81-84, 86-88]. Second, as discussed above, as the dual epidemics of obesity and T2D progress, glucose intolerance and atherogenic dyslipidemia follow, with increases in levels of TG, TRL, and cholesterol remnants. Greater characterization of these dyslipidemias prior to n-3 PUFA may assist in defining subpopulations with greater residual risk that could benefit the most from n-3 PUFA interventions. Third, more research needs to focus upon the optimum dose and forms of n-3 PUFA in various contexts since some investigators have reported greater response rates with higher doses of n-3 PUFA [91, 102-105].

\section{Conclusion}

In recent reports, imperfections in prior n-3 PUFA studies included nonlinear and decreasing reported death rates, variations in the background of n-3 PUFA intake, heterogeneity in study designs, dose, rapidity, and duration of administration, purity, peroxidation, and adulterating products, underpowering, short follow-up periods, disparate findings, variation in disease substrates, individual responsiveness and adherence, difficulties comparing component studies in meta-analyses, and confounding by cotherapies.

The available evidence tends to refute the impression that improving n-3 PUFA stores has little utility in the current era of cardiology care. Rather, for those interested in avoiding vascular damage and preventing the need for these therapies, $n-3$ PUFA may provide a relatively inexpensive, beneficial adjunctive approach. Optimal amounts for the general population are unknown, and given the heterogeneity in responsiveness, it may be impossible, even after performing blood level tests, to answer this question in a particular individual.

Even so, there are now many large-scale studies showing benefits, including the most recent high-quality metaanalyses discussed above. n-3 PUFA are highly pleiotropic agents with many documented physiological effects. Concordance between preclinical, observational RCTs and meta-analyses is impressive. These agents have mod- 
est, statistically significant benefits, which may accrue over time. In all systematic reviews and meta-analyses, the selection of component studies influences outcomes. This overarching principle must be incorporated into a synthesis of the totality of data, particularly when evidence is inconsistent or conflicting. Reasons for inconsistencies between studies are still incompletely understood, and gaps in knowledge are evident. This report has included several sources of evidence that inform practice. In addition, combined with the impressive safety profile, the reward/risk ratio of n-3 PUFA is extremely high.

Some authorities prefer to favor n-3 PUFA consumption in the form of fish, but many bodies, including the American Heart Association, advise caution because of the mercury and other chemical pollutants present in the fish supply [106]. Indeed, although restriction of fish intake other than perhaps for pregnant/breastfeeding women is not universally advised, serious questions remain unanswered and are not inconsequential in $\mathrm{CV}$ patients [107-109].
Based on the totality of evidence, physicians and public health agencies should encourage the attainment of recommended amounts of n-3 PUFA in the population and, in particular, optimum levels in CV patients. Effect sizes in selected secondary prevention populations range up to an $18 \%$ reduction in $\mathrm{RR}$, likely depending upon the lipid profile. There is a suggestion that effect sizes may vary with baseline $\mathrm{n}-3$ levels. In this respect, it is agreed that even potential reductions in CHD deaths of $10 \%$ "would justify treatment" of n-3 PUFA administration due to the favorable safety profile $[87,91,99,100]$.

Studies in progress may provide further welcome information. In the interim, considering n-3 PUFA consumption as an intervention associated with moderate outcome improvements appears to be reasonable, particularly using the AHA Advisory recommendations [99]. Optimal use of n-3 PUFA requires individualization according to pathophysiology and biomarkers, and is yet another reminder that medical practice remains both an art and a science.

\section{References}

1 Kones R, Rumana U: Current treatment of dyslipidaemia: a new paradigm for statin drug use and the need for additional therapies. Drugs Springer 2015;75:1187-1199.

2 Benjamin E, Blaha M, Chiuve S, et al: Heart disease and stroke statistics - 2017 update: a report from the American Heart Association. Circulation 2017;135:e146-e603.

3 Kones R: Molecular sources of residual cardiovascular risk, clinical signals, and innovative solutions: relationship with subclinical disease, undertreatment, and poor adherence: implications of new evidence upon optimizing cardiovascular patient outcomes. Vasc Health Risk Manag 2013;9:617-670.

4 Global Organization of EPA and DHA Omega-3S: GOED publishes report on omega-3 finished products. 2016. http://www.goedomega3.com//news/viewnews/56 (accessed September 17, 2017).

5 Sinclair $\mathrm{H}$ : The diet of Canadian Indians and Eskimos. Proc Nutr Soc 1953;12:69-82.

6 Bang H, Dyerberg J: Lipid metabolism and ischemic heart disease in Greenland Eskimos. Adv Nutr Res 1980;3:1-22.

7 Kromhout D, Bosschieter E, de Lezenne-Coulander C: The inverse relation between fish consumption and 20-year mortality from coronary heart disease. N Engl J Med 1985;312: 1205-1209.

8 Albert C, Hennekens C, O'Donnell C, et al: Fish consumption and risk of sudden cardiac death. JAMA 1998;279:23-28.
9 Albert C, Campos H, Stamper M, et al: Blood levels of long-chain n-3 fatty acids and the risk of sudden death. N Engl J Med 2002;346: 1113-1118.

10 Harris W, Poston W, Haddock C: Tissue n-3 and n-6 fatty acids and risk for coronary heart disease events. Atherosclerosis 2007;193:110.

11 Lavie C, Milani R, Mehra M, et al: Omega-3 polyunsaturated fatty acids and cardiovascular diseases. J Am Coll Cardiol 2009;54:585594.

12 DeCaterina R: n-3 fatty acids in cardiovascular disease. N Engl J Med 2011;364:24392450 .

13 Russo G: Dietary n-6 and n-3 polyunsaturated fatty acids: from biochemistry to clinical implications in cardiovascular prevention. Biochem Pharm 2009;77:937-946.

14 Harris W, Jacobson T: Omega-3 fatty acids; in Ballantyne CM (ed): Clinical Lipidology: A Companion to Braunwald's Heart Disease. Philadelphia, WB Saunders, 2009, pp 326338.

15 Bays H, Tighe A, Sadovsky R, et al: Prescription omega-3 fatty acids and their lipid effects: physiologic mechanisms of action and clinical implications. Expert Rev Cardiovasc Ther 2008;6:391-409.

16 Harris W: The omega-3 index as a risk factor for coronary heart disease. Am J Clin Nutr 2008;87:1997S-2002S

17 Mozaffarian D, Rimm E: Fish intake, contaminants, and human health: evaluating the risks and the benefits. JAMA 2006;296:1885-1899.
18 Kris-Etherton P, Harris W, Appel L, et al American Heart Association Nutrition Committee: Fish consumption, fish oil, omega-3 fatty acids, and cardiovascular disease. Circulation 2002;106:2747-2757.

19 Rizos E, Ntzani E, Bika E, et al: Association between $\omega$-3 fatty acid supplementation and risk of major cardiovascular disease events: a systematic review and meta-analysis. JAMA 2012;308:1024-1033.

20 von Schacky C: Omega-3 fatty acids in cardiovascular disease - an uphill battle. Prostaglandins Leukot Essent Fat Acids 2015;92:41-47.

21 Albert B, Cameron-Smith D, Garg M, et al: Marine oils: complex, confusing, confounded? J Nutr Intermed Metab 2016;5:3-10.

22 Mori T, Woodman R: The independent effects of eicosapentaenoic acid and docosahexaenoic acid on cardiovascular risk factors in humans. Curr Opin Clin Nutr Metab Care 2006;9:95-104.

23 Stark K, van Elswyk M, Higgins M, et al: Global survey of the omega-3 fatty acids, docosahexaenoic acid and eicosapentaenoic acid in the blood stream of healthy adults. Prog Lipid Res 2016;63:132-152.

24 von Schacky C: Omega-3 index and cardiovascular health. Nutrients 2014;6:799-814.

25 Shaikh SR: Biophysical and biochemical mechanisms by which dietary N-3 polyunsaturated fatty acids from fish oil disrupt membrane lipid rafts. J Nutr Biochem 2012;23: 101-105. 
26 Turk HF, Chapkin RS: Membrane lipid raft organization is uniquely modified by $n-3$ polyunsaturated fatty acids. Prostaglandins Leukot Essent Fatty Acids 2013;88:43-47.

27 Hou TY, McMurray DN, Chapkin RS: Omega-3 fatty acids, lipid rafts, and T cell signaling. Eur J Pharmacol 2016;785:2-9.

28 Dart C: Lipid microdomains and the regulation of ion channel function. J Physiol 2010 588:3169-3178

29 Farag NE, Jeong D, Claydon T, et al: Polyunsaturated fatty acids inhibit $\mathrm{K}_{\mathrm{v}} 1.4$ by interacting with positively charged extracellular pore residues. Am J Physiol Cell Physiol 2016; 311:C255-C268.

30 Moreno C, de la Cruz A, Oliveras A, et al: Marine n-3 PUFAs modulate IKs gating, channel expression, and location in membrane microdomains. Cardiovasc Res 2015;105:223-232.

31 Gillet L, Roger S, Bougnoux P, et al: Beneficial effects of omega-3 long-chain fatty acids in breast cancer and cardiovascular diseases: voltage-gated sodium channels as a common feature? Biochimie 2011;93:4-6.

32 Li G-R, Sun H-Y, Zhang X-H, et al: Omega-3 polyunsaturated fatty acids inhibit transient outward and ultra-rapid delayed rectifier $\mathrm{K}^{+}$ currents and $\mathrm{Na}^{+}$current in human atrial $\mathrm{my}-$ ocytes. Cardiovasc Res 2009;81:286-293.

33 Xiao YF, Sigg DC, Leaf A: The antiarrhythmic effect of n-3 polyunsaturated fatty acids: modulation of cardiac ion channels as a potential mechanism. J Membr Biol 2005;206: 141-154.

34 Billman G: The effects of omega-3 polyunsaturated fatty acids on cardiac rhythm: a critical reassessment. Pharmacol Ther 2013;140:5380.

35 Moreno C, Macias A, Prieto A, et al: Polyunsaturated fatty acids modify the gating of $\mathrm{Kv}$ channels. Front Pharmacol 2012;3:163.

36 Dujardin K, Dumotier B, David M, et al: Ultrafast sodium channel block by dietary fish oil prevents dofetilide-induced ventricular arrhythmias in rabbit hearts. Am J Physiol Heart Circ Physiol 2008;295:H1414-H1421.

37 Den Ruijter H, Berecki G, Opthof T, et al: Proand antiarrhythmic properties of a diet rich in fish oil. Cardiovasc Res 2007;73:316-325.

38 Coronel R: The pro- or antiarrhythmic actions of polyunsaturated fatty acids and of cholesterol. Pharmacol Ther 2017;176:40-47.

39 Moreno C, Macías A, Prieto A, et al: Effects of $\mathrm{n}-3$ polyunsaturated fatty acids on cardiac ion channels. Front Pharmacol 2012;9:245.

40 Bharadwaj S, Gohel T, Deen O, et al: Fish oilbased lipid emulsion: current updates on a promising novel therapy for the management of parenteral nutrition-associated liver disease. Gastroenterol Rep Oxf 2015;3:110-114.

41 Lands B: Benefit-risk assessment of fish oil in preventing cardiovascular disease. Drug Saf 2016;39:787-799.

42 Marchioli R, Barzi F, Bomba E, et al; GISSIPrevenzione Investigators: Early protection against sudden death by $n-3$ polyunsaturated fatty acids after myocardial infarction: time- course analysis of the results of the Gruppo Italiano per lo Studio della Sopravvivenza nell'Infarto Miocardico (GISSI)-Prevenzione. Circulation 2002;105:1897-1903.

43 Julian D, Camm A, Frangin G, et al: Randomised trial of effect of amiodarone on mortality in patients with left-ventricular dysfunction after recent myocardial infarction: EMIAT. Lancet 1997;349:667-674.

44 Kones R, Rumana U: Nutrition studies, optimum cardiovascular health, and coronary atherosclerosis - ensuring a solid foundation for public policy. J Clin Exp Cardiol 2016;7:e145.

45 Moss J, Ramji D: Nutraceutical therapies for atherosclerosis. Nat Rev Cardiol 2016;13: 513-532.

46 Marik P, Varon J: Omega-3 dietary supplements and the risk of cardiovascular events: a systematic review. Clin Cardiol 2009;32:365372.

47 Foran J, Good D, Carpenter D, et al: Quantitative analysis of the benefits and risks of consuming farmed and wild salmon. J Nutr 2005; 135:2639-2643.

48 He K, Song Y, Daviglus M, et al: Fish consumption and incidence of stroke: a metaanalysis of cohort studies. Stroke 2004;35: 1538-1542.

49 He K, Song Y, Daviglus M, et al: Accumulated evidence on fish consumption and coronary heart disease mortality: a meta-analysis of cohort studies. Circulation 2004;109:27052711.

50 Hooper L, Thompson R, Harrison R, et al: Omega 3 fatty acids for prevention and treatment of cardiovascular disease. Cochrane $\mathrm{Da}$ tabase Syst Rev 2004;4:CD003177.

51 Hooper L, Thompson R, Harrison R, et al: Risks and benefits of omega 3 fats for mortality, cardiovascular disease, and cancer: systematic review. BMJ 2006;332:752-760.

52 Wang C, Harris W, Chung M, et al: n-3 fatty acids from fish or fish-oil supplements, but not $\alpha$-linolenic acid, benefit cardiovascular disease outcomes in primary- and secondaryprevention studies: a systematic review. Am J Clin Nutr 2006;84:5-17.

53 Leon H, Shibata M, Sivakumaran S, et al: Effect of fish oil on arrhythmias and mortality: systematic review. BMJ 2008;337:a2931.

54 Mente A, de Koning L, Shannon H, et al: A systematic review of the evidence supporting a causal link between dietary factors and coronary heart disease. Arch Intern Med 2009; 169:659-769.

55 Brouwer I, Raitt M, Dullemeijer C, et al: Effect of fish oil on ventricular tachyarrhythmia in three studies in patients with implantable cardioverter defibrillators. Eur Heart J 2009;30: $820-826$

56 Harris W, Mozaffarian D, Lefevre M, et al: Towards establishing dietary reference intakes for eicosapentaenoic and docosahexaenoic acids. J Nutr 2009;139:804S-819S.

57 Writing Group for the AREDS2 Research Group, Bonds D, Harrington M, Worrall B, et al: Effect of long-chain $\omega-3$ fatty acids and lu- tein + zeaxanthin supplements on cardiovascular outcomes: results of the Age-Related Eye Disease Study 2 (AREDS2) randomized clinical trial. JAMA Intern Med 2014;174: 763-771.

58 Mozaffarian D, Wu J: Omega-3 fatty acids and cardiovascular disease: effects on risk factors, molecular pathways, and clinical events. J Am Coll Cardiol 2011;58:2047-2067.

59 Burr M, Fehily A, Gilbert J, et al: Effects of changes in fat, fish, and fibre intakes on death and myocardial reinfarction: diet and reinfarction trial (DART). Lancet 1989;2:757761

60 GISSI-Prevenzione Investigators: Dietary supplementation with n-3 polyunsaturated fatty acids and vitamin $\mathrm{E}$ after myocardial infarction: results of the GISSI-Prevenzione trial. Lancet 1999;354:447-455.

61 Burr M, Ashfield-Watt P, Dunstan F, et al: Lack of benefit of dietary advice to men with angina: results of a controlled trial. Eur J Clin Nutr 2003;57:193-200.

62 Yokoyama M, Origasa H, Matsuzaki M, et al Japan EPA Lipid Intervention Study (JELIS) Investigators: Effects of eicosapentaenoic acid on major coronary events in hypercholesterolaemic patients (JELIS): a randomised openlabel, blinded endpoint analysis. Lancet 2007; 369:1090-1098.

63 Tavazzi L, Maggioni A, Marchioli R, et al GISSI-HF Investigators: Effect of $n-3$ polyunsaturated fatty acids in patients with chronic heart failure (the GISSI-HF trial): a randomised, double-blind, placebo-controlled trial. Lancet 2008;372:1223-1230.

64 Rauch B, Schiele R, Schneider S, et al; OMEGA Study Group: OMEGA, a randomized, placebo-controlled trial to test the effect of highly purified omega- 3 fatty acids on top of modern guideline-adjusted therapy after myocardial infarction. Circulation 2010;122: 2152-2159.

65 Galan P, Kesse-Guyot E, Czernichow S, et al: Effects of B vitamins and omega 3 fatty acids on cardiovascular diseases: a randomised placebo controlled trial. BMJ 2013;341:c6273.

66 The Risk and Prevention Study Collaborative Group: $\mathrm{n}-3$ fatty acids in patients with multiple cardiovascular risk factors. N Engl J Med 2013;368:1800-1808.

67 Archer E, Pavela G, Lavie C: The inadmissibility of what we eat in America and NHANES dietary data in nutrition and obesity research and the scientific formulation of national dietary guidelines. Mayo Clin Proc 2015;90: 911-926.

68 Duda M, O’Shea K, Tintinu A, et al: Fish oil, but not flaxseed oil, decreases inflammation and prevents pressure overload-induced cardiac dysfunction. Cardiovasc Res 2009;81: 319-327.

69 Le Grand B: Effects of Omacor ${ }^{\circledR}$ on left ventricular remodelling consecutive to post myocardial infarction special issue - myocardial infarction. World J Cardiovasc Dis 2013;3: $40-44$. 
70 Nodari S, Triggiani M, Campia U, et al: Effects of $n-3$ polyunsaturated fatty acids on left ventricular function and functional capacity in patients with dilated cardiomyopathy. J Am Coll Cardiol 2011;57:870-879.

71 Tang W, Samara M: Polyunsaturated fatty acids in heart failure: should we give more and give earlier? J Am Coll Cardiol 2011;57:880883.

72 Yancy C, Jessup M, Bozkurt B, et al; American College of Cardiology Foundation/American Heart Association Task Force on Practice Guidelines: 2013 ACCF/AHA guideline for the management of heart failure: a report of the American College of Cardiology Foundation/American Heart Association Task Force on practice guidelines. Circulation 2013; 128:e240-e327.

73 Xin W, Wei W, Li X: Effects of fish oil supplementation on cardiac function in chronic heart failure: a meta-analysis of randomised controlled trials. Heart 2012;98:1620-1625.

74 Ramadeen A, Laurent G, dos Santos C, et al: n-3 polyunsaturated fatty acids alter expression of fibrotic and hypertrophic genes in a dog model of atrial cardiomyopathy. Heart Rhythm 2010;7:520-528.

75 Zanetti M, Grillo A, Losurdo P, et al: Omega-3 polyunsaturated fatty acids: structural and functional effects on the vascular wall. Biomed Res 2015;2015:791978.

76 Merino J, Sala-Vila A, Kones R, et al: Increasing long-chain n-3 PUFA consumption improves small peripheral artery function in patients at intermediate-high cardiovascular risk. J Nutr Biochem 2014;25:642-646.

77 van den Elsen L, Spijkers L, van den Akker R, et al: Dietary fish oil improves endothelial function and lowers blood pressure via suppression of sphingolipid-mediated contractions in spontaneously hypertensive rats. J Hypertens 2014;32:1050-1058.

78 Kones R: Inflammation, CRP, and cardiometabolic risk: how compelling is the potential therapeutic role of omega-3 PUFA in cardiovascular disease? Clin Lipidol 2011;6:627630.

79 Massaro M, Scoditti E, Carluccio M, et al: Omega-3 fatty acids, inflammation and angiogenesis: basic mechanisms behind the cardioprotective effects of fish and fish oils. Cell Mol Biol 2010;56:59-82.

80 Wang C, Xiong B, Huang J: The role of omega-3 polyunsaturated fatty acids in heart failure: a meta-analysis of randomised controlled trials. Nutrients 2017;1:18

81 Schuchardt J, Hahn A: Bioavailability of longchain omega-3 fatty acids. Prostaglandins Leukot Essent Fat Acids 2013;89:1-8.

82 Mason R, Sherratt S: Omega-3 fatty acid fish oil dietary supplements contain saturated fats and oxidized lipids that may interfere with their intended biological benefits. Biochem Biophy Res Commun 2017;483:425-429.
83 Joensen A, Overvad K, Dethlefsen C, et al: Marine n-3 polyunsaturated fatty acids in adipose tissue and the risk of acute coronary syndrome. Circulation 2011;124:1232-1238.

84 Block R, Harris W, Reid K, et al: EPA and DHA in blood cell membranes from acute coronary syndrome patients and controls. Atherosclerosis 2008;197:821-828.

85 Yagi S, Aihara K, Fukada D, et al: Reduced ratio of eicosapentaenoic acid and docosahexaenoic acid to arachidonic acid is associated with early onset of acute coronary syndrome. Nutr J 2015;14:111.

86 Harris W, Pottala J, Lacey S, et al: Clinical correlates and heritability of erythrocyte eicosapentaenoic and docosahexaenoic acid content in the Framingham Heart Study. Atherosclerosis 2012;225:425-431.

87 Del Gobbo L, Imamura F, Aslibekyan S, et al; Cohorts for Heart and Aging Research in $\mathrm{Ge}$ nomic Epidemiology (CHARGE), Fatty Acids and Outcomes Research Consortium (FORCE): $\omega-3$ polyunsaturated fatty acid biomarkers and coronary heart disease: pooling project of 19 cohort studies. JAMA Intern Med 2016;176:1-13.

88 Harnack K, Andersen G, Somoza V: Quantitation of $\alpha$-linolenic acid elongation to eicosapentaenoic and docosahexaenoic acid as affected by the ratio of $n 6 / n 3$ fatty acids. Nutr Metab 2009;6:8.

89 Simopoulos A, DiNicolantonio J: The importance of a balanced $\omega-6$ to $\omega-3$ ratio in the prevention and management of obesity. Open Heart 2016;3:e000385.

90 Wang D, Li Y, Chiuve S, et al: Association of specific dietary fats with total and cause-specific mortality. JAMA Intern Med 2016;176: 1134-1145.

91 Alexander D, Miller P, van Elswyk M, et al: A meta-analysis of randomized trials and prospective cohort studies of eicosapentaenoic and docosahexaenoic long chain omega-3 fatty acids and coronary heart disease risk. Mayo Clin Proc 2017;92:15-29.

92 Nordestgaard B, Varbo A: Triglycerides and cardiovascular disease. Lancet 2014;384:626635.

93 Nordestgaard B: Triglyceride-rich lipoproteins and atherosclerotic cardiovascular disease: new insights from epidemiology, genetics, and biology. Circ Res 2016;118:547-563.

94 Varbo A, Benn M, Tybjærg-Hansen A, et al: Elevated remnant cholesterol causes both low-grade inflammation and ischemic heart disease, whereas elevated low-density lipoprotein cholesterol causes ischemic heart disease without inflammation. Circulation 2013 128:1298-1309.

95 Varbo A, Benn M, Smith G, et al: Remnant cholesterol, low-density lipoprotein cholesterol, and blood pressure as mediators from obesity to ischemic heart disease. Circ Res 2015; 116:665-673.
96 Toth P: Triglyceride-rich lipoproteins as a causal factor for cardiovascular disease. Vasc Health Risk Manag 2016;12:171-183.

97 Ridker P: Residual inflammatory risk: addressing the obverse side of the atherosclerosis prevention coin. Eur Heart J 2016;37: 1720-1722.

98 Ridker P: How common is residual inflammatory risk? Circ Res 2017;120:617-619.

99 Siscovick D, Barringer T, Fretts A, et al: Omega-3 polyunsaturated fatty acid (fish oil) supplementation and the prevention of clinical cardiovascular disease: a science advisory from the American Heart Association. Circulation 2017;135:e1-e18.

100 Maki K, Palacios O, Bell M, et al: Use of supplemental long-chain omega-3 fatty acids and risk for cardiac death: an updated metaanalysis and review of research gaps. J Clin Lipidol 2017;11:1152-1160.e2.

101 O'Keefe J, Jacob D, Lavie C: Omega-3 fatty acid therapy: the tide turns for a fish story. Mayo Clin Proc 2017;92:1-3.

102 Flock M, Skulas-Ray A, Harris W, et al: Determinants of erythrocyte omega-3 fatty acid content in response to fish oil supplementation: a dose-response randomized controlled trial. J Am Heart Assoc 2013;2: e000513.

103 Itakura H, Yokoyama M, Matsuzaki M, et al Relationships between plasma fatty acid composition and coronary artery disease. J Atheroscler Thromb 2011;18:99-107.

104 Mozaffarian D, Lemaitre R, King I, et al Plasma phospholipid long-chain omega-3 fatty acids and total and cause-specific mortality in older adults: a cohort study. Ann Intern Med 2013;158:515-525.

105 Heydari B, Abdullah S, Pottala J, et al: Effect of omega-3 acid ethyl esters on left ventricular remodeling after acute myocardial infarction: the OMEGA-REMODEL Randomized Clinical Trial. Circulation 2016; 134:378-391.

106 Cusack L, Smit E, Kile M, et al: Regional and temporal trends in blood mercury concentrations and fish consumption in women of child bearing age in the United States using NHANES data from 1999-2010. Environ Health 2017;16:10.

107 Kim K, Kabir E, Jahan S: A review on the distribution of $\mathrm{Hg}$ in the environment and its human health impacts. J Hazard Mater 2016;306:376-385.

108 Perkins J, Petriello M, Newsome B, et al: Polychlorinated biphenyls and links to cardiovascular disease. Env Sci Pollut Res Int 2016;23:2160-2172.

109 Rizzo G, Baroni L: Health and ecological implications of fish consumption: a deeper insight. Med J Nutr Metab 2016;9:7-22. 